\title{
CP Phenomenology in Heterotic String Models
}

\author{
Oleg Lebedev
}

Centre for Theoretical Physics, University of Sussex, Brighton BN1 9QJ, UK

\begin{abstract}
I discuss aspects of the CP problem in heterotic orbifolds which arises in models with low energy supersymmetry.
\end{abstract}

Recent measurements of the CP asymmetry in the $B \rightarrow \Psi K_{s}$ decay [1] imply that CP is significantly violated in nature and is not an approximate symmetry. Supersymmetric models with large CP phases generally predict fermion electric dipole moments (EDMs) that far exceed the experimental limits [2], and this presents a major challenge for low energy supersymmetry, the so called SUSY CP problem (see [3] for a review).

In supergravity models, the soft supersymmetry breaking terms are functions of the hidden sector fields which enter the superpotential and the Kähler potential. In particular, the trilinear parameters are written as [4]

$$
A_{\alpha \beta \gamma}=F^{m}\left[\hat{K}_{m}+\partial_{m} \log Y_{\alpha \beta \gamma}-\partial_{m} \log \left(\tilde{K}_{\alpha} \tilde{K}_{\beta} \tilde{K}_{\gamma}\right)\right] .
$$

Here the Latin indices refer to the hidden sector fields while the Greek indices refer to the observable fields; the Kähler potential is expanded in observable fields as $K=$ $\hat{K}+\tilde{K}_{\alpha}\left|C^{\alpha}\right|^{2}+\ldots$ and $\hat{K}_{m} \equiv \partial_{m} \hat{K}$. The sum in $m$ runs over all of the SUSY breaking fields. We note that the supergravity notation for the A-terms and Yukawas can be connected to the "usual" one by fixing the order of the indices as follows: the first index is to refer to the Higgs fields, the second to the quark doublets, and the third to the quark singlets, e.g. $Y_{H_{1} Q_{i} D_{j}} \equiv Y_{i j}^{d}$.

It is clear that if the auxiliary fields $F^{m}$ are complex, the A-terms and other soft breaking terms will have complex phases. Such flavor-independent complex phases induce large EDMs, in conflict with experiment. This is the well known SUSY CP problem.

This problem may seem to be circumvented by assuming that the F-terms are real. However, this is not case [5],[7]. First, let us note that in order to generate the Yukawa couplings hierarchy, these couplings must be functions of some scalar fields (e.g. moduli). Thus, the corresponding derivatives in (1) do not vanish. Now, if the auxiliary partners of these scalars break supersymmetry, e.g. $F^{m} \neq 0$, the resulting A-terms are non-universal or flavor-dependent. As a result, the soft trilinear parameters $A_{\alpha \beta \gamma} Y_{\alpha \beta \gamma}$ are not diagonalized by the same transformations as the Yukawa matrices. So, in the physical (super-CKM) basis, the A-terms are not diagonal and their diagonal entries contain complex phases which result from diagonalization of the complex Yukawa matrices:

$$
\hat{U}_{L, R} \rightarrow V_{L, R}^{u} \hat{U}_{L, R} \quad, \quad \hat{D}_{L, R} \rightarrow V_{L, R}^{d} \hat{D}_{L, R}
$$




$$
\begin{aligned}
& Y^{u} \rightarrow V_{L}^{u T} Y^{u} V_{R}^{u *}=\operatorname{diag}\left(h_{u}, h_{c}, h_{t}\right), \\
& Y^{d} \rightarrow V_{L}^{d T} Y^{d} V_{R}^{d *}=\operatorname{diag}\left(h_{d}, h_{s}, h_{b}\right) .
\end{aligned}
$$

The strength of this effect can be estimated in the case of heterotic orbifolds. The matter fields must belong to the twisted sectors since otherwise the couplings are 1 or 0 and the mass hierarchy cannot be generated at the renormalizable level. The Yukawa coupling of the states at the fixed points $f_{1,2,3}$ belonging to the twisted sectors $\theta_{1,2,3}$ is given by $[6]$

$$
Y_{f_{1} f_{2} f_{3}}=N \sum_{u \in Z^{n}} \exp \left[-4 \pi T\left(f_{23}+u\right)^{T} M\left(f_{23}+u\right)\right]
$$

where $f_{23} \equiv f_{2}-f_{3}, N$ is a normalization factor, and the matrix $M$ (with fractional entries) is related to the internal metric of the orbifold. Here $T$ is normalized such that $T \rightarrow T+i$ is a symmetry of the Lagrangian. For a realistic case, $\operatorname{Re} T=\mathcal{O}(1)$, the sum is dominated by one term and

$$
\partial_{T} \ln Y_{f_{1} f_{2} f_{3}} \simeq-4 \pi f_{23}^{T} M f_{23}
$$

for some (typically fractional) $f_{23}$ depending on the positions of the fixed points. Unless $F^{T}$ is suppressed, this creates an order one non-universality in the A-terms which results in large EDMs (see [7] for numerical examples). In addition, this effect induces flavor changing neutral currents (FCNC). However the FCNC are not particularly sensitive to the flavor structure of the A-terms, so such effects are only subleading.

This observation implies that either the flavor structure of the Yukawa matrices or the pattern of SUSY breaking is quite special. One possibility is that the supersymmetry breaking is dilaton-dominated. In this case, only $F^{S}$ is non-zero and the dangerous flavor effects disappear. To see whether this situation is possible in heterotic models, we analyze the vacua as functions of the dilaton $S$ and an overall modulus $T$. To stabilize the dilaton, we use the non-perturbative Kähler potential

$$
K_{S}=\ln \left(\frac{1}{2 \operatorname{Re} S}+d(\operatorname{Re} S)^{\frac{p}{2}} e^{-b \sqrt{\operatorname{Re} S}}\right),
$$

where $d, p, b$ are certain constants $(p, b>0)$. To produce CP violation in the Yukawa couplings, e.g. to achieve a complex $T$ at the minimum of the scalar potential, we multiply the standard gaugino condensate superpotential by a modular invariant function

$$
H(T)=[j(T)-1728]^{\frac{m}{2}} j(T)^{\frac{n}{3}} P[j(T)],
$$

where $j(T)$ is the absolutely modular invariant function, $P[j(T)]$ is some polynomial of $j(T)$, and $m, n$ are some negative fractional numbers. The results of the minimization of the scalar potential [8] are given in Table 1.

We see that with appropriate $m, n$ dilaton dominated SUSY breaking is possible. The corresponding SUSY parameters are flavor blind and the unwanted flavor mixing is absent.

An interesting feature of this set-up is an axionic symmetry $S \rightarrow S+i \alpha$ with a real continuous $\alpha$. This means that the phase of the dilaton field is unphysical and $S$ can 


\begin{tabular}{|c||c|c|c||}
\hline$\delta_{G S}$ & 0 & 1 & 1.5 \\
\hline \hline$m$ & $-\frac{1}{15}$ & $-\frac{1}{30}$ & $-\frac{1}{90}$ \\
$n$ & $-\frac{2}{15}$ & $-\frac{2}{30}$ & $-\frac{2}{90}$ \\
$S_{\min }$ & 1.75 & 1.71 & 1.69 \\
$T_{\min }$ & $1.38+0.36 i$ & $1.45+0.44 i$ & $1.33+0.33 i$ \\
$\varphi_{\mathrm{CKM}}$ & $\mathcal{O}(1)$ & $\mathcal{O}(1)$ & $\mathcal{O}(1)$ \\
$V_{0} / M_{\mathrm{Pl}}^{4}$ & $1.32 \times 10^{-32}$ & $1.28 \times 10^{-32}$ & $1.42 \times 10^{-32}$ \\
$F_{S} / \mathrm{Gev}$ & -2150 & -2120 & -2230 \\
$F_{T} / \mathrm{Gev}$ & $\sim 0$ & $\sim 0$ & $\sim 0$ \\
$M_{a} / \mathrm{Gev}$ & -604 & -608 & -646 \\
$m_{\alpha} / \mathrm{Gev}$ & 280 & 276 & 290 \\
$A_{\alpha \beta \gamma} / \mathrm{Gev}$ & 1690 & 1660 & 1750 \\
$\hat{\mu} / \mathrm{Gev}$ & 1.75 & 1.73 & 1.82 \\
$\sqrt{B \hat{\mu}} / \mathrm{Gev}$ & 280 & 276 & 290 \\
\hline \multicolumn{2}{r|}{}
\end{tabular}

Table 1: Minima and SUSY breaking parameters.

be made real by the symmetry transformation. The resulting $F^{S}$ is also real and the supersymmetric CP phases are absent. At the same time, $T$ has an order one phase and is away from the fixed points of the modular group, so an order one CKM phase can be produced [9]. Thus, in this scenario, CP violation appears in the Standard Model sector but not in the supersymmetric sector. One however should remember that the symmetry $S \rightarrow S+i \alpha$ is only perturbative and the space-time non-perturbative effects may induce small SUSY CP phases (in the case of a single gaugino condensate).

The soft breaking parameters of Table 1 are not fully realistic since the $\mu$-term is too small and there are color breaking minima. It is also worth noting that the cosmological constant $V_{0}$ is non-zero. However, $V_{0}$ coincides with the cosmological constant only at the tree level, so that inclusion of the quadratically divergent supergravity corrections changes this picture drastically and $V_{0}$ and the cosmological constant become independent parameters [10]. Radiative corrections also have a significant effect on the $\mu$-term and other parameters, so the viability of the model can be analyzed only after incorporation of the quantum effects.

To summarize, I have discussed certain aspects of the "string" CP problem and presented a semi-realistic example how this problem can be circumvented.

\section{References}

[1] B. Aubert et al., Phys. Rev. Lett. 87, 091801 (2001).

[2] M.V. Romalis, W.C. Griffith, and E.N. Fortson, Phys. Rev. Lett. 86, 2505 (2001); P.G. Harris et al., Phys. Rev. Lett. 82 (1999), 904. 
[3] S. Abel, S. Khalil, and O. Lebedev, Nucl. Phys. B 606, 151 (2001).

[4] S. K. Soni and H. A. Weldon, Phys. Lett. B 126 (1983) 215; V. S. Kaplunovsky and J. Louis, Phys. Lett. B 306 (1993) 269; A. Brignole, L. E. Ibanez, and C. Munoz, hep-ph/9707209.

[5] S. Khalil, O. Lebedev, and S. Morris, Phys. Rev. D 65, 115014 (2002).

[6] J. A. Casas, F. Gomez, and C. Munoz, Int. J. Mod. Phys. A 8, 455 (1993); D. Bailin, G. V. Kraniotis, and A. Love, Phys. Lett. B 435, 323 (1998).

[7] S. Abel, S. Khalil, and O. Lebedev, hep-ph/0112260.

[8] O. Lebedev and S. Morris, hep-th/0203246.

[9] O. Lebedev, Phys. Lett. B 521, 71 (2001).

[10] K. Choi, J. E. Kim, and H. P. Nilles, Phys. Rev. Lett. 73, 1758 (1994). 